\title{
Understanding the effects of electronic polarization and delocalization on charge- transport levels in oligoacene systems
}

Christopher Sutton, Naga Rajesh Tummala, Travis Kemper, Saadullah G. Aziz, John Sears, Veaceslav

Coropceanu, and Jean-Luc Brédas

Citation: The Journal of Chemical Physics 146, 224705 (2017); doi: 10.1063/1.4984783

View online: http://dx.doi.org/10.1063/1.4984783

View Table of Contents: http://aip.scitation.org/toc/jcp/146/22

Published by the American Institute of Physics

\section{Articles you may be interested in}

Analytic derivative couplings and first-principles exciton/phonon coupling constants for an ab initio FrenkelDavydov exciton model: Theory, implementation, and application to compute triplet exciton mobility parameters for crystalline tetracene

The Journal of Chemical Physics 146, 224110 (2017); 10.1063/1.4985607

Confirming the relationship between first hyperpolarizability and the bond length alternation coordinate for merocyanine dyes

The Journal of Chemical Physics 146, 224505 (2017); 10.1063/1.4985672

Effect of the diradical character on static polarizabilities and two-photon absorption cross sections: A closer look with spin-flip equation-of-motion coupled-cluster singles and doubles method

The Journal of Chemical Physics 146, 224103 (2017); 10.1063/1.4984822

Perspective: Theory and simulation of hybrid halide perovskites

The Journal of Chemical Physics 146, 220901 (2017); 10.1063/1.4984964

Hard-hard coupling assisted anomalous magnetoresistance effect in amine-ended single-molecule magnetic junction

The Journal of Chemical Physics 146, 224701 (2017); 10.1063/1.4984821

Laplace-transformed multi-reference second-order perturbation theories in the atomic and active molecular orbital basis

The Journal of Chemical Physics 146, 224101 (2017); 10.1063/1.4984591

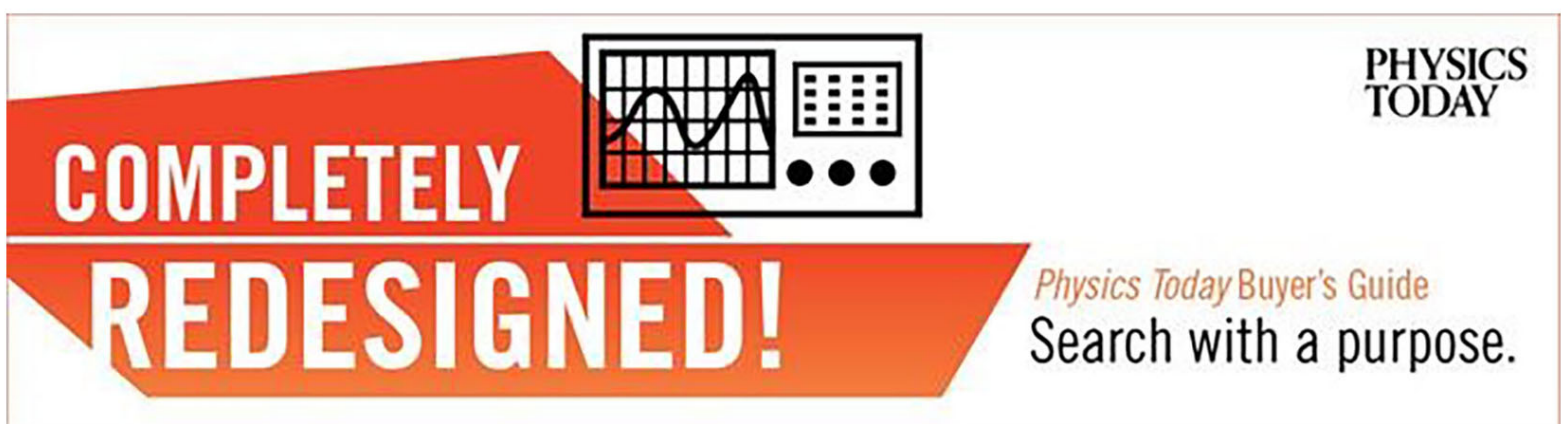




\title{
Understanding the effects of electronic polarization and delocalization on charge-transport levels in oligoacene systems
}

\author{
Christopher Sutton, ${ }^{1, a)}$ Naga Rajesh Tummala, ${ }^{1, a)}$ Travis Kemper, ${ }^{1}$ Saadullah G. Aziz, ${ }^{2}$ \\ John Sears, ${ }^{1}$ Veaceslav Coropceanu, ${ }^{1, b)}$ and Jean-Luc Brédas ${ }^{1,3, b)}$ \\ ${ }^{1}$ School of Chemistry and Biochemistry and Center for Organic Photonics and Electronics, \\ Georgia Institute of Technology, Atlanta, Georgia 30332-0400, USA \\ ${ }^{2}$ Department of Chemistry, King Abdulaziz University, Jeddah 21589, Saudi Arabia \\ ${ }^{3}$ Laboratory for Computational and Theoretical Chemistry of Advanced Materials, Division of Physical Science \\ and Engineering, King Abdullah University of Science and Technology, Thuwal 23955-6900, \\ Saudi Arabia
}

(Received 30 March 2017; accepted 18 May 2017; published online 13 June 2017)

\begin{abstract}
Electronic polarization and charge delocalization are important aspects that affect the charge-transport levels in organic materials. Here, using a quantum mechanical/embedded-charge (QM/EC) approach based on a combination of the long-range corrected $\omega$ B97X-D exchange-correlation functional (QM) and charge model 5 (CM5) point-charge model (EC), we evaluate the vertical detachment energies and polarization energies of various sizes of crystalline and amorphous anionic oligoacene clusters. Our results indicate that $\mathrm{QM} / \mathrm{EC}$ calculations yield vertical detachment energies and polarization energies that compare well with the experimental values obtained from ultraviolet photoemission spectroscopy measurements. In order to understand the effect of charge delocalization on the transport levels, we considered crystalline naphthalene systems with QM regions including one or five-molecules. The results for these systems show that the delocalization and polarization effects are additive; therefore, allowing for electron delocalization by increasing the size of the QM region leads to the additional stabilization of the transport levels. Published by AIP Publishing. [http://dx.doi.org/10.1063/1.4984783]
\end{abstract}

\section{INTRODUCTION}

A comprehensive understanding of the electronic structure of organic molecular semiconductors is critical for the development of accurate charge-injection (or extraction) and charge-transport models. The charge-transport levels are defined by the ionization potential (IP) and electron affinity (EA) of the individual molecules. Because of inter-molecular interactions in the bulk environment, electron delocalization and electronic polarization of the surrounding molecules act to modulate the isolated-molecule energy levels.

The polarization energy arises from the interaction of a charge carrier appearing on a given molecule with the induced and permanent multipole moments of the neighboring molecules. ${ }^{1}$ Various methods to treat electronic polarization in crystalline systems have been detailed in recent review articles. $^{2,3}$ Most of these approaches aim to provide the correct description of the electrostatic potential related to a molecule. This is achieved by using distributed (static) multipoles (e.g., in micro-electrostatic models ${ }^{4,5}$ or polarizable force-field based models) ${ }^{6,7}$ from a quantum mechanical (QM) calculation, in conjunction with atomic polarizabilities to describe the dynamic polarizability contributions. Other approaches rely on QM or semi-empirical methods to evaluate the correct response to the presence of the excess charge in the polarizable

\footnotetext{
a) C. Sutton and N. R. Tummala contributed equally to this work.

b) Authors to whom correspondence should be addressed: coropceanu@ gatech.edu and jean-luc.bredas@chemistry.gaetch.edu
}

region [e.g., in quantum mechanical/molecular mechanics $(\mathrm{QM} / \mathrm{MM}),{ }^{8,9}$ Valence-Bond/Hartree-Fock $(\mathrm{VB} / \mathrm{HF}),{ }^{10}$ or charge redistribution $(\mathrm{CR})^{5}$ based models]. While these methods aim at accurately describing the polarization energy, modeling charge injection (extraction) and charge transport in organic materials also requires an understanding of the evolution of the transport levels that, in addition to electronic polarization, are affected by factors such as charge delocalization and surface dipole. ${ }^{11}$

Experimentally, the IP and EA of a material are most often measured with ultraviolet photoemission spectroscopy (UPS) and inverse photoemission spectroscopy (IPES), respectively. ${ }^{12-14}$ The EA of a molecular cluster can be also estimated by means of UPS measurements on a negatively charged system. Such an approach has been applied to naphthalene, anthracene, and tetracene clusters and is of particular interest to our study. ${ }^{15-18}$ In these investigations, anionic molecular clusters are first produced by electron impact ionization and the ground state of these negatively charged systems is subsequently probed using UPS. The experimental data suggest that the excess charge in some anionic clusters can delocalize over multiple molecules. In addition, the interpretation of the recent experimental results by Yoshida $e t a l$. for pentacene and perfluoropentacene thin films points to IP and EA values changing when going from an isolated molecule to bulk due to the additive effect of electronic polarization and electronic delocalization. ${ }^{19}$ In contrast, a very recent GW-based $\mathrm{QM} / \mathrm{MM}$ study on pentacene by Li et $^{3}{ }^{3}$ indicates that allowing for charge delocalization (by increasing the $\mathrm{QM}$ region and 
(a)<smiles>c1ccc2ccccc2c1</smiles>

(b)<smiles>c1ccc2cc3ccccc3cc2c1</smiles>

(c)<smiles>c1ccc2cc3cc4ccccc4cc3cc2c1</smiles>

(d)<smiles>c1ccc2cc3cc4cc5ccccc5cc4cc3cc2c1</smiles>

FIG. 1. Molecular structures of the linear oligoacenes investigated in this work: (a) naphthalene, (b) anthracene, (c) tetracene, and (d) pentacene. decreasing the polarizable region) has no effect on the transport gap and electron and hole transport levels. These contradictory results indicate that there is a need for a better understanding of the effects of electronic polarization and carrier delocalization on the transport levels.

Regarding the QM modeling of charge delocalization in a material, it was shown previously that the application of standard (semi)local density functional theory (DFT) functionals to the description of EAs in small molecules can be challenging due to the instability of anions coming from the self-interaction error. $^{20,21}$ Therefore, a robust methodology, capable of describing adequately both charge (de)localization and that can properly treat the electronic polarization, is required. Here, we apply a QM/EC (embedded-charge) approach based on the combination of the long-range corrected $\omega$ B97X-D exchangecorrelation functional and charge model 5 (CM5) point-charge model $^{22}$ to evaluate the electron affinity and polarization energy in finite anionic clusters of naphthalene, including two- and three-dimensional clusters of various sizes. We then compare the polarization energies in clusters of naphthalene, anthracene, tetracene, and pentacene (see Fig. 1) kept in their crystalline configurations. Finally, in order to gain insight into the interplay between charge localization/delocalization and electronic polarization and their impact on the transport levels, we compare the results derived for model naphthalene systems in which the QM description is applied to one-molecule and five-molecule clusters.

\section{METHODOLOGY}

In the framework of standard DFT, the incorrect exponential decay of the approximate exchange-correlation functionals leads to spurious charge delocalization, ${ }^{23-25}$ which has been shown to strongly affect the description of an excess charge in crystalline oligoacenes. ${ }^{21}$ Therefore, here, we have chosen to employ an approach based on a system-dependent, non-empirically tuned long-range corrected functional, which has been demonstrated to dramatically reduce the delocalization error present in standard DFT methods. ${ }^{26-28}$ Specifically, we use the $\omega \mathrm{B} 97 \mathrm{X}-\mathrm{D}$ functional, ${ }^{29}$ with the range-separation parameter $\omega$ partitioning the Coulomb operator into shortrange and long-range components. The $\omega$ value is tuned by minimizing the difference between the highest occupied molecular orbital (HOMO) eigenvalue of the ground state and the IP, with the latter being the difference between the ground-state energies of the neutral molecule and the cation,
$E_{g s}(\omega, N)-E_{g s}(\omega, N-1)$ (a procedure referred to as IPtuning). ${ }^{30-33}$ We note that the IP-tuning is considered here as opposed to tuning to the electron affinity because naphthalene does not have a stable anionic state (vide infra). ${ }^{34}$

In terms of structures, the molecular geometries of the crystalline systems and lattice parameters were taken from the Cambridge Structural Database. ${ }^{35-37}$ For the amorphous clusters, molecular dynamics (MD) simulations of oligoacenes were performed with the OPLS-AA (optimized potential for liquid simulations-all atom) force field parameters ${ }^{38}$ using GROMACS 4.6.2. ${ }^{39}$ The long-range electrostatic interactions were computed using the particle mesh Ewald method with an accuracy of $10^{-5} .40$ The initial configuration consisted of placing 1000 molecules within a simulation box at a density lower than $0.3 \mathrm{~g} / \mathrm{cc}$. An NVT (constant number of molecules, constant volume, and constant temperature) ensemble simulation was then conducted at $1000 \mathrm{~K}$ for $1 \mathrm{~ns}$, followed by a NPT (constant number of molecules, constant pressure, and constant temperature) simulation for a total of $10 \mathrm{~ns}$, of which the first 5 ns were used to gradually decrease the temperature from $1000 \mathrm{~K}$ to $300 \mathrm{~K}$ with the next $5 \mathrm{~ns}$ at $300 \mathrm{~K}$. The average density over the last $2 \mathrm{~ns}$ of the NPT simulation is $1.10 \mathrm{~g} / \mathrm{cc}$.

We evaluated the electronic polarization of an excess charge through a self-consistent QM/EC calculation. The QM/EC procedure consists of iterative DFT single-point computations for each of the molecules in the cluster in the presence of atom-centered point charges representing the surrounding molecules. These charges are computed using the CM5 charge model, ${ }^{22,41,42}$ which is applicable to both charged and neutral molecules. In addition, the CM5 model is known to exhibit only a small dependence on the choice of a basis set and functional. $^{22,41,42}$ The iterative procedure starts with a DFT single-point calculation on the central molecule, while using the atom-centered point charges for the surrounding molecules in the cluster obtained from the DFT calculations on the isolated molecules. The DFT calculations are then performed consecutively for each molecule in the cluster by updating the atom-centered point charges on other molecules from previous DFT runs. This self-consistent procedure is continued until the change in the total energy of the central molecule is less than $10^{-4}$ hartree $(2.7 \mathrm{meV})$ between two iterations. ${ }^{8}$ When evaluating anionic clusters, the DFT calculations are performed with an extra electron on the molecule in the center of the cluster. Various cluster sizes varying from 5 to 225 molecules, in two-dimensional and three-dimensional packings, are 
investigated for naphthalene; cluster sizes of $\sim 60-70$ molecules are considered for anthracene, tetracene, and pentacene.

To evaluate charge delocalization, we have calculated the charge distribution of a five-molecule cluster using the IP-tuned $\omega$ B97X-D methodology with the $6-31+G(d, p)^{43}$ basis set. For comparison and validation, we also computed the charge distribution using a complete-active space selfconsistent field (CASSCF)/6-31G(d) approach. All calculations were performed with Gaussian 09 (Rev. D). ${ }^{44}$

At this stage, we believe it is helpful to define some of the key properties that are examined in this study. The polarization energy related to the presence of an excess electron is defined as

$$
P^{-}=E A_{B}-E A_{G},
$$

where $E A_{G}$ and $E A_{B}$ are the electron affinities of the isolated molecule in the gas phase and of the crystalline bulk, respectively. When intra- and inter-molecular structural relaxation effects are considered, there are three possible descriptions of the electron affinity (the same applies to the ionization potential, which is relevant for the case of an excess positive charge): the vertical electron affinity (vEA), the adiabatic electron affinity (aEA), and the vertical detachment energy (vDE). These quantities are defined as

$$
\begin{aligned}
v E A & =E_{\text {neutral }}^{o}-E_{\text {neutral }}^{-}, \\
a E A & =E_{\text {neutral }}^{o}-E_{\text {anion }}^{-}, \\
v D E & =E_{\text {anion }}^{o}-E_{\text {anion }}^{-},
\end{aligned}
$$

where $E_{\text {neutral }}^{o}$ is the energy of the neutral system at its equilibrium geometry; $E_{\text {neutral }}^{-}$is the radical-anion energy at the optimized neutral geometry; $E_{\text {anion }}^{o}$ is the energy of the neutral system at the optimized radical-anion geometry; and $E_{\text {anion }}^{-}$is the radical-anion energy at its optimized radical-anion geometry. The energies determined from UPS and IPES are measured according to the adiabatic principle, i.e., at the optimal geometry of the initial state. Since, in the UPS studies of oligoacene clusters mentioned above, ${ }^{15,17,18}$ an anionic molecular cluster is first produced, we assume that these experiments probe the geometrically and electronically relaxed anionic clusters and their results can be used to estimate the vDE values (and polarization energies) in these systems.

The polarization energies were evaluated according to Eq. (1) by computing $E A_{G}$ and $E A_{B}$ both at the total $\triangle \mathrm{SCF}$ (self-consistent field) level using the total energies of the neutral and anion states. We also computed these properties in the framework of Koopmans' theorem (KT), where the EAs of the clusters are approximated by the HOMO eigenvalues of the radical-anion states. Only the intramolecular geometry relaxation of the charged molecule was considered in our calculations. We note, however, that previous studies have shown that in both crystalline and amorphous organic molecular systems, the external contribution to the relaxation energy is much smaller than the intramolecular counterpart. ${ }^{45,46}$

\section{RESULTS AND DISCUSSION}

\section{A. Single-molecule detachment energies}

The computed and experimental vDE values for the isolated molecules of naphthalene, anthracene, tetracene, and pentacene are in very good agreement (differences of
TABLE I. IP-tuned $\omega$ B97X-D/6-31+G(d,p) vEAs at the crystalline geometry (indicated by ${ }^{\mathrm{a}}$ ), and geometry-optimized single-molecule vEAs and vDEs for naphthalene, anthracene, tetracene, and pentacene compared with experiment. All values are in $\mathrm{eV}$. Error bars for the experimental values are estimated to be $0.05 \mathrm{eV}$ from the references.

\begin{tabular}{lrrrc}
\hline \hline & \multicolumn{3}{c}{ IP-tuned $\omega B$ 97X-D } & \multirow{2}{*}{ Exp. } \\
\cline { 2 - 4 } System & \multicolumn{1}{c}{ vEA $^{\mathrm{a}}$} & \multicolumn{1}{c}{ vEA } & vDE & vDE \\
\hline Naphthalene & -0.49 & -0.51 & -0.20 & $-0.19^{\mathrm{b}}$ \\
Anthracene & 0.19 & 0.29 & 0.53 & $0.53-0.54^{\mathrm{c}}$ \\
Tetracene & 0.88 & 0.85 & 1.07 & $1.04-1.06^{\mathrm{d}}$ \\
Pentacene & 1.11 & 1.23 & 1.41 & $1.35^{\mathrm{e}}$ \\
\hline \hline
\end{tabular}

${ }^{\mathrm{a} C a l c u l a t i o n s}$ performed at the crystalline geometry.

${ }^{\mathrm{b}}$ Reference 34 .

${ }^{c}$ References 15,17 and 47.

${ }^{\mathrm{d}}$ References 15 and 48 .

${ }^{\mathrm{e}}$ Reference 48 .

$<0.06 \mathrm{eV}$ ), see Table I. Taking the advantage of the fact that the IP-tuned $\omega \mathrm{B} 97 \mathrm{X}-\mathrm{D} / 6-31+\mathrm{G}(\mathrm{d}, \mathrm{p})$ methodology results in an accurate description of single-molecule vDEs, we next apply the methodology to naphthalene clusters in various packing configurations and then compare our results to similarly sized anthracene, tetracene, and pentacene clusters.

\section{B. Two-dimensional (2-D) clusters of naphthalene}

We begin with a discussion of a 5-molecule 2-D naphthalene cluster, in which the excess electron is localized on the central molecule. The central naphthalene molecule and the four closest surrounding molecules are in the herringbone arrangement (located in $a b$ plane, see Fig. 2). The anion is unstable (i.e., the vDE is negative) and the polarization energy $\left(P^{-}\right)$computed at the $\triangle \mathrm{SCF}$ level is nearly zero, see

(a)

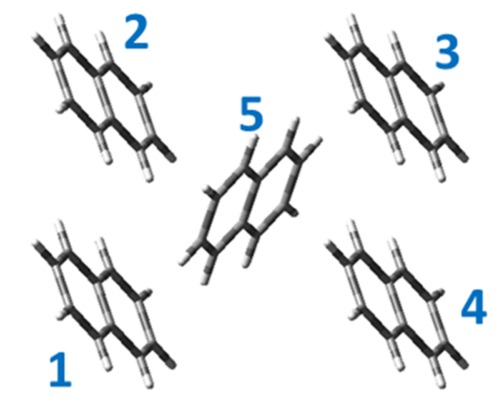

(b)

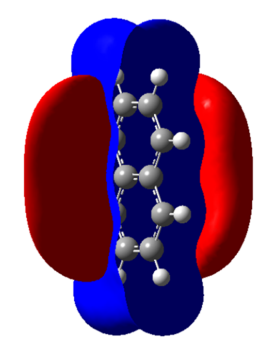

(c)

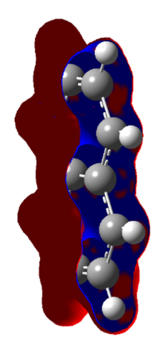

FIG. 2. (a) A crystalline structure of a five-naphthalene cluster along the $a b$ plane; the $c$-axis of the crystal would be protruding in and out of the paper. Similar configurations were used to model anthracene, tetracene, and pentacene clusters. [(b) and (c)] Electrostatic potentials based on electron density for the neutral and anionic naphthalene molecule, respectively, based on the geometry in the crystal structure is used to plot the electrostatic potential using Gaussview. ${ }^{49}$ The blue and red colors represent positive and negative electrostatic potential values of 0.01 atomic units. 
TABLE II. Results of QM/EC calculations on naphthalene 2-D and 3-D clusters; the QM region includes a single molecule. The 3-D clusters (with their radius indicated) are extracted from the crystal structure of naphthalene. The vDE values are computed in the crystal structure geometry and corrected with the relaxation energy of the charged molecule. All values are in $\mathrm{eV}$.

\begin{tabular}{|c|c|c|c|}
\hline Radius ( $\AA$ ) (number of molecules) & vDE & $P^{-}(\triangle \mathrm{SCF})$ & $P^{-}(\mathrm{KT})$ \\
\hline $0(1)$ & -0.20 & $\ldots$ & \\
\hline $2-D \ldots(5)$ & -0.19 & 0.01 & 0.07 \\
\hline 2-D 10 (13) & 0.01 & 0.21 & 0.30 \\
\hline 2-D 22 (63) & -0.04 & 0.16 & 0.29 \\
\hline \multicolumn{4}{|c|}{ Crystalline (3-D) } \\
\hline $12(47)$ & 0.43 & 0.63 & 0.87 \\
\hline $17(116)$ & 0.49 & 0.69 & 0.97 \\
\hline \multicolumn{4}{|c|}{ Amorphous (3-D) } \\
\hline $8(13)$ & 0.73 & 0.93 & 1.15 \\
\hline $10(22)$ & 0.80 & 1.00 & 1.29 \\
\hline $12(35)$ & 0.84 & 1.04 & 1.34 \\
\hline $15(70)$ & 0.91 & 1.11 & 1.46 \\
\hline
\end{tabular}

Table II, indicating that the stabilization coming from the inplane first shell of molecules is negligible. When increasing the size of the 2-D cluster to $n=13$ (where $n$ is the number of molecules, Fig. S1 of the supplementary material) the polarization energy increases by $0.20 \mathrm{eV}$. However, interestingly, the computed vDE is then only ca. $+0.01 \mathrm{eV}$; thus, the anion is barely stabilized by the in-plane neighboring molecules within the herringbone configuration when extended beyond the first shell. Further increasing the 2-D cluster to $n=63$ (Fig. S2 of the supplementary material) decreases the polarization energy by $0.05 \mathrm{eV}\left(P^{-}=0.16 \mathrm{eV}\right)$, while the $\mathrm{vDE}$ becomes $-0.04 \mathrm{eV}$ and thus the anion is unstable. Our results for the 2-D crystalline clusters compare well with the polarization energy of $\sim 0.25 \mathrm{eV}$ previously reported from 2-D anthracene clusters ranging from $n=20$ to $\mathrm{n}=150$ using a self-consistent approach within the valence bond theory at the Austin Model 1 (AM1) level. ${ }^{10}$ Overall, the results underline that, within a 2-D naphthalene cluster, the polarization energy is not sufficient to stabilize the anionic charge. It is worth noting that the $\triangle \mathrm{SCF}$ and Koopman's theorem (KT) approaches yield similar trends for the polarization energies; however, the absolute values are slightly different.

The inspection of the electrostatic potential obtained for naphthalene (see Fig. 2) shows a large negative potential perpendicular to the molecular plane and parallel to the molecular long axis (shown in red color in Fig. 2) and a positive potential in the plane of the molecule. Therefore, an overall repulsive in-plane interaction is expected for the 2-D clusters in the herringbone packing because of the high density of interacting negative (repulsive) electrostatic potentials between neutral and anion (red colored regions in Fig. 2). Rearrangement of the electron density will lead to an energetic stabilization; however, the induced-dipole interactions in the 2-D clusters are not sufficiently stabilizing to offset the repulsive interactions from the like charges. Thus, our results indicate that to stabilize an excess electron in naphthalene, the presence of attractive (positive potential) interlayer interactions is required. In Sec. III C, we thus turn to the 3-D clusters.

\section{Three-dimensional (3-D) clusters of naphthalene}

The 3-D cluster configurations (Figs. S3-S5 in the supplementary material) are obtained by extending the size of the 2-D layers and adding extra layers on both sides. The addition of one extra molecular layer on each side, which completely encapsulates the central charged molecule (see Fig. S3 in the supplementary material), results in a significant increase in the polarization energy and for a stable vDE of $+0.43 \mathrm{eV}$ for the $n=473$-D cluster. Intermediate configurations that provide for incomplete encapsulation result in smaller stabilization energies. Thus, in a 3-D cluster of $n=25$ molecules (see Fig. S4 in the supplementary material), the $\mathrm{vDE}$ is only about $+0.36 \mathrm{eV}$. The addition of two more layers, i.e., going to a 5-layer cluster with $n=116$ (see Fig. S5 in the supplementary material), increases the vDE slightly to a value of $+0.49 \mathrm{eV}$. The vDE calculations for a 3-layer cluster with $n=116$ molecules yield a value of $+0.42 \mathrm{eV}$, i.e., nearly the same as that obtained for the 3-layer cluster of $n=47$, including that the increase of $0.06 \mathrm{eV}$ in the 5-layer cluster is due to additional fourth and fifth layers. We note that the experimental vDEs attributed to crystalline clusters show a step-increase from $0.86 \mathrm{eV}$ to $1.1 \mathrm{eV}$ when going from $n=47$ to $n>$ ca. $70 .{ }^{18}$ This step increase in the experimental vDE value suggests that upon increasing beyond a certain size threshold, the cluster configuration changes to one with more layers.

We note that the estimated polarization energy for a $n=116$ five-layer cluster with a centrally localized charge accounts for ca. two-thirds of the experimental value $(+1.10$ $\mathrm{eV}$ ) for the naphthalene single crystal. ${ }^{1}$ The polarization energy is expected to continue to increase with the cluster size, especially upon addition of interlayer molecules. Nevertheless, the major contributions are captured within the first few shells of neighboring molecules.

\section{Amorphous clusters of naphthalene}

It must be borne in mind that we are comparing our results with experimental data on clusters for which geometric structures are in fact unknown. Hence, for the sake of consistency, we have also investigated disordered clusters (see Figs. S6S9 in the supplementary material) where the excess electron is localized on the central naphthalene molecule. For the smallest cluster examined ( $n=13$ molecules), a vDE value of $+0.73 \mathrm{eV}$ is determined, which is more than twice what is calculated for a similarly sized ordered cluster. The vertical detachment energies and polarization energies are calculated to increase with the cluster size; however, when compared to experiment, ${ }^{50}$ the calculated vDE values remain lower, even for the largest cluster sizes considered. This could be related to the fact that intermolecular geometry relaxations are not considered explicitly upon addition of the excess charge to the amorphous clusters. In contrast to the crystalline clusters, we find here a linear increase in the $\mathrm{vDE}$ as a function of $\mathrm{N}^{-1 / 3}$ (where $\mathrm{N}$ is the cluster size). This result is consistent with the observed evolution in disordered clusters. ${ }^{18}$ This increase in the vDE is anticipated to saturate and plateau at very large cluster sizes. We note that the polarization energy derived from Koopman's theorem $\left(P^{-}\right)$ 
is consistently ca. $0.2-0.3 \mathrm{eV}$ higher than $P^{-}$obtained at the $\triangle \mathrm{SCF}$ level $\left[P^{-}(\triangle \mathrm{SCF})\right]$ and increases with the cluster size, in contrast to the 2-D case.

For an $n=70$ amorphous cluster, the computed vDE $(+0.91 \mathrm{eV})$ and $P^{-}(\Delta \mathrm{SCF})(+1.11 \mathrm{eV})$ are $0.5 \mathrm{eV}$ larger than what was computed for the crystalline cluster of the same size (Table II). This $0.5 \mathrm{eV}$ difference in the polarization energy derived from the $\mathrm{QM} / \mathrm{EC}$ calculations is in close agreement with the experimental value $(\sim 0.4 \mathrm{eV}$, estimated from the spectra reported in Ref. 18). The larger polarization energy can be attributed to a re-orientation of the "in-plane" molecules, which we recall do not stabilize the extra electron in the ordered system. These confirm that packing and orientation can affect the polarization energy, as was recently reported for pentacene thin films on silica and graphite ${ }^{19}$ and from a comparison of in $P^{+}$values in pentacene and 6,13-bis(triisopropylsilylethinyl)pentacene. ${ }^{51}$ Given the qualitatively correct trends observed for ordered and disordered clusters of naphthalene, we now compare the electronic polarization energies in similarly sized clusters of anthracene, tetracene, and pentacene.

\section{E. Detachment energies in clusters of anthracene, tetracene, and pentacene}

For the $n=71$ crystalline naphthalene cluster, there occurs a difference of $0.6 \mathrm{eV}$ between the vDE computed at the QM/EC level and that observed experimentally. ${ }^{18}$ Going to anthracene and tetracene (to the best of our knowledge, no experimental results have been reported for pentacene), smaller deviations $(0.2-0.3 \mathrm{eV})$ are found between the computed and experimental vDE values, see Table III. For similarsize clusters with three molecular layers in each configuration (i.e., cluster radii around 15-16 $\AA$ ), the computed polarization energies represent about $69 \%, 77 \%$, and $57 \%$ of the experimental values for bulk anthracene, tetracene, and pentacene, respectively. Some disagreement among these values is expected because our calculations consider small, finite clusters. These results indicate that the calculated polarization energies for the excess electron in the clusters, although qualitatively follows in the right trends, are consistently too low, possibly because of the limited sizes we were able to consider in this study. In addition to the fact that we do not take into account the geometry relaxation of the lattice (surrounding

TABLE III. Results of QM/EC calculations for similarly sized clusters of naphthalene, anthracene, tetracene, and pentacene, where the $\mathrm{QM}$ region includes a single molecule and the radius of the 3-D cluster extracted from the crystal structure ranging from 14.5 to $16.5 \AA$. All values are in $\mathrm{eV}$.

\begin{tabular}{lccccccc}
\hline \hline \multirow{2}{*}{$\begin{array}{l}\text { Molecule (number } \\
\text { of molecules) }\end{array}$} & \multicolumn{3}{c}{ IP-tuned $\omega$ BD97X-D } & & \multicolumn{2}{c}{ Exp. } \\
\cline { 2 - 4 } \cline { 7 - 8 } & vDE & $P^{-}(\Delta \mathrm{SCF})$ & $P^{-}(\mathrm{KT})$ & & vDE $(n=70)$ & $P^{-}($bulk $)$ \\
\hline Naphthalene (71) & 0.42 & 0.62 & 0.88 & & $1.0 / 1.4^{\mathrm{a}}$ & $1.10^{\mathrm{b}}$ \\
Anthracene (69) & 1.12 & 0.75 & 0.83 & & $1.3 / 1.5^{\mathrm{c}}$ & $1.09^{\mathrm{b}}$ \\
Tetracene (71) & 1.50 & 0.71 & 0.80 & & $1.8 / 2.0^{\mathrm{d}}$ & $0.92^{\mathrm{d}}$ \\
Pentacene (59) & 1.92 & 0.64 & 0.74 & & $\cdots$ & $1.17^{\mathrm{b}}$ \\
\hline \hline
\end{tabular}

${ }^{\mathrm{a}}$ Reference 18 .

${ }^{\mathrm{b}}$ Reference 1 .

${ }^{\mathrm{c}}$ Reference 15 .

${ }^{\mathrm{d}}$ Reference 15 . molecules), another factor is the possible delocalization of the excess electron, to which we now turn in Sec. III F.

\section{F. Effect of charge delocalization}

In the discussed above calculations, the excess charge in the radical-anion state of the clusters was kept localized on the central molecule. However, in crystalline materials, some extent of delocalization of the charge carriers over a finite number of molecules can be expected. In fact, a high degree of delocalization corresponding to band transport rather than polaron transport has been suggested for a number of highly purified organic single crystals, such as oligoacenes and derivatives. $^{52,53}$ For instance, angle-resolved photoelectron spectroscopy measurements have recently revealed a bandlike dispersion in the highest-occupied state of pentacene and rubrene. ${ }^{54-56}$ Charge delocalization was also suggested to take place in anionic oligoacene clusters. ${ }^{15,17,18}$

Since the QM treatment of large clusters is computationally demanding, we have extended our studies on naphthalene by treating at the QM level a cluster of five molecules [see Fig. 2(a)]. The CM5 atomic charges derived from the IP-tuned $\omega$ B97X-D calculations are shown in Table IV. In order to check the reliability of the $\omega \mathrm{B} 97 \mathrm{X}-\mathrm{D}$ results, we also computed the charge distribution at the CAS-SCF level, a methodology that allows an understanding of the potentially multi-reference nature of the wavefunction. Both approaches indicate that about $70 \%$ of the excess charge remains localized on the central molecule. However, some difference is observed between the delocalization patterns of the remaining $30 \%$ of the excess charge. The CAS-SCF charge distribution is almost symmetric, as opposed to what is seen for the IP-tuned $\omega \mathrm{B} 97 \mathrm{X}-\mathrm{D}$ charges. Overall, however, the calculations indicate that the IP-tuned $\omega$ B97X-D functional does provide a reliable degree of charge delocalization.

The transport levels of a molecule embedded in a medium or crystal environment are stabilized by the electronic polarization that arises from the interaction of a localized charge carrier with the induced and permanent multipole moments of the surrounding molecules. However, when the excess charge delocalizes over several sites, there appears an additional contribution to the stabilization energy due to the resonance energy (band dispersion) and a change in electrostatic interactions due to the redistribution of the excess charge density. For the sake of simplicity, we refer to this additional component

TABLE IV. CAS-SCF $(11,10) / 6-31 \mathrm{G}(\mathrm{d})$ and IP-tuned $\omega \mathrm{B} 97 \mathrm{X}-\mathrm{D} / 6-$ $31+\mathrm{G}(\mathrm{d}, \mathrm{p})$ calculated total CM5 charge distribution for each molecule in Fig. 2 for the lowest energy anion state in the herringbone configuration of five naphthalene clusters.

\begin{tabular}{lcc}
\hline \hline & \multicolumn{2}{c}{ Naphthalene } \\
\cline { 2 - 3 } Molecule & $\omega B$ 97X-D & CAS-SCF \\
\hline 1 & -0.04 & -0.07 \\
2 & -0.09 & -0.07 \\
3 & -0.04 & -0.07 \\
4 & -0.09 & -0.07 \\
5 & -0.74 & -0.72 \\
Total & -1.00 & -1.00 \\
\hline \hline
\end{tabular}


TABLE V. The stabilization energy, polarization energy (from Table II), and delocalization energy for various naphthalene clusters with QM regions, including one or five molecules, calculated at the KT and $\triangle \mathrm{SCF}$ levels. All values are in $\mathrm{eV}$.

\begin{tabular}{|c|c|c|c|c|c|c|}
\hline \multirow[t]{2}{*}{$\begin{array}{l}\text { System (number } \\
\text { of molecules) }\end{array}$} & \multicolumn{2}{|c|}{$\begin{array}{c}\text { Stabilization energy } \\
(\mathrm{eV})\end{array}$} & \multicolumn{2}{|c|}{$\begin{array}{c}\text { Polarization energy } \\
(\mathrm{eV})\end{array}$} & \multicolumn{2}{|c|}{$\begin{array}{c}\text { Delocalization energy } \\
(\mathrm{eV})\end{array}$} \\
\hline & KT & $\triangle \mathrm{SCF}$ & KT & $\triangle \mathrm{SCF}$ & KT & $\triangle \mathrm{SCF}$ \\
\hline Naphthalene (5) & 0.41 & 0.16 & 0.07 & 0.01 & 0.34 & 0.15 \\
\hline \multicolumn{7}{|c|}{ Naphthalene cluster with the central five molecules treated at the QM level } \\
\hline $12 \AA(47)$ & 1.19 & 0.79 & 0.87 & 0.63 & 0.32 & 0.16 \\
\hline $22.5 \AA(116)$ & 1.28 & 0.85 & 0.97 & 0.69 & 0.32 & 0.16 \\
\hline
\end{tabular}

as the delocalization contribution. However, it is not obvious how to separate the polarization and delocalization contributions. Therefore, here, we take a practical view and define the delocalization contribution as the difference between the stabilization energy computed for a cluster with the excess charge allowed to delocalize over five molecules and the stabilization energy calculated for the same cluster with the excess charge localized on a single molecule. In other words, we subtract from the overall stabilization energies the corresponding electronic polarization energies computed using a localized charge in Sec. III C.

For a five-molecule herringbone naphthalene cluster [Fig. 2(a)], the stabilization energy derived in the framework of Koopmans' theorem (i.e., the difference between the HOMO energy of the five-molecule system and the HOMO energy of an isolated molecule both computed at the QM level) is +0.41 $\mathrm{eV}$, see Table $\mathrm{V}$. This value is about $+0.16 \mathrm{eV}$ at the $\triangle \mathrm{SCF}$ level, which is obtained by the difference in the total energies of the charged and neutral five-molecule cluster. Based on these values for the stabilization energy and the values for the polarization energy taken from Table II, the delocalization energy is estimated to be $0.34 \mathrm{eV}$ and $0.15 \mathrm{eV}$ at the $\mathrm{KT}$ and $\triangle \mathrm{SCF}$ levels, respectively. The results indicate that, in contrast to $\mathrm{QM} / \mathrm{EC}$ calculations discussed above, the radical-anion state of a five-molecule naphthalene cluster is nearly stable when electron delocalization is taken into account.

We have also considered larger 3-D clusters containing $n=47$ and 116 molecules [see Figs. S3 and S5 of the supplementary material) while now treating the central five molecules at the QM level. In comparison with the fivemolecule cluster, the stabilization increases by $+0.78(+0.87)$ $\mathrm{eV}$ ] when going from the $n=5$ to the $n=47(n=116)$ cluster at the KT level. In comparison, a stabilization of +0.63 $(+0.69) \mathrm{eV}$ is computed when using the $\triangle \mathrm{SCF}$ energies. At the KT level, the estimated delocalization energy is $0.32 \mathrm{eV}$ for both 3-D clusters and compares very well with the 0.34 $\mathrm{eV}$ value obtained for the five-molecule cluster. Similarly, the $\triangle \mathrm{SCF}$ calculations provide a delocalization energy of $\sim 0.16$ $\mathrm{eV}$ for the larger 3-D clusters, which is comparable to that observed for the 2-D 5-molecule cluster $(+0.15 \mathrm{eV})$. Similar values of the delocalization energy at all cluster sizes are not surprising since the size of the QM region was the same in all calculations.

Overall, our results indicate that an increase in the QM region from one to five molecules leads to an increase in the stabilization of the electron transport level and EA energy of the naphthalene clusters. As discussed above, the stabilization energy includes both the polarization energy and the delocalization energy. In order to estimate the polarization around the delocalized anion, the stabilization energy of the five-molecule clusters is subtracted from the $n=47(n=116)$ cluster, which results in a polarization energy $+0.63(0.69)$ $\mathrm{eV}$. This value is identical to the estimated polarization energy in Table II for a localized charge on a single molecule for a similarly sized cluster. Therefore, these results indicate that the polarization energy around the delocalized anion does not change. This feature is attributed to the fact that the intra-plane contribution to the polarization energy is much smaller than the inter-plane contribution, and thus, the delocalization and polarization become additive (which we label as the stabilization energy).

Although a different material was examined, our finding contrasts qualitatively with the recent $\mathrm{QM} / \mathrm{MM}$ results $^{3}$ from Li et al. for pentacene, which indicate that an increase in the QM region from one to three and five molecules leads to a reduction in the polarization energy. In the work of $\mathrm{Li}$ et al., ${ }^{3}$ the increase in the delocalization contribution resulting from the increase in the QM region appears to be compensated by a decrease in electronic-polarization contribution. In our work, the additional four molecules included in the QM region in the case of our calculations contribute to the delocalization energy but do not impact the electronic polarization energy (see Table II). These discrepancies might arise from the particular choices of the QM regions in the two studies and our consideration here of only the electron transport level rather than the band gap. Further investigations of how charge delocalization and electronic polarization affect the transport levels are thus desirable.

\section{CONCLUSIONS}

In this work, we use a QM/EC approach based on a combination of long-range corrected DFT with the CM5 point-charge model to describe the electron affinity and the polarization energy in oligoacene systems. The results indicate that the $\mathrm{QM} / \mathrm{EC}$ calculations can qualitatively reproduce the experimental detachment energies of finite naphthalene clusters derived by means of UPS measurements.

The results from calculations of 2-D naphthalene clusters with the excess electron localized on the central molecule suggest that in-plane interactions do not significantly 
contribute to the stabilization of the anion, especially in the finite clusters studied. Our QM/EC computational approach points to the out-of-plane dipoles (interlayer interactions) as are the major contributors to the stability of the excess charge.

For 3-D clusters of naphthalene, interestingly, we find that the peak experimentally attributed to the crystalline-like structure is due to a 3-D cluster that appears when a localized charge is encapsulated completely with the addition of extra layers to the central 2-D herringbone packing layer. This occurs for a cluster size of ca. $n=47$ molecules and the additional layers along the c-axis increase the polarization energy that evolves in a step-wise manner in crystalline clusters. Calculations on finite clusters for a series of oligoacenes ranging from $n=59-71$ molecules yield polarization energies that are larger than $50 \%$ of the bulk values even without the consideration of the electron delocalization.

For disordered 3-D clusters, in line with experimental data, we observe a linear increase in vertical detachment energies (vDEs) and polarization energies. Our results also show that both quantities are significantly larger in such amorphous clusters when compared to the similarly sized crystalline clusters.

Finally, in order to understand the effect of charge delocalization on the transport levels, we considered crystalline naphthalene systems with a central five-molecule herringbone region treated at the quantum level. The results from CAS-SCF and IP-tuned $\omega$ B97X-D calculations show that $30 \%$ of the excess electron is delocalized on the additional four molecules included in the QM region. A comparison of the results obtained for similarly sized systems, with QM regions of one and five molecules, allowed for a quantification of the effect that the charge delocalization has on the stabilization energy of the charged cluster; this contribution was computed at $\triangle \mathrm{SCF}$ (KT) level to be ca. $0.15(0.3) \mathrm{eV}$ in addition to the polarization energy. Overall, these results underline that when ordered systems are considered both delocalization and polarization effects should be taken into account. However, more investigations along these lines are clearly needed.

\section{SUPPLEMENTARY MATERIAL}

See supplementary material for the illustrations of the various 2-D and 3-D clusters employed in this work.

\section{ACKNOWLEDGMENTS}

We gratefully acknowledge the financial support for this work at the Georgia Institute of Technology by the Deanship of Scientific Research of King Abdulaziz University under an International Collaboration Grant (Award No. D-001-433), the National Science Foundation through its MRSEC program (Award No. DMR-0819885), and by King Abdullah University of Science and Technology (V.C.). We also acknowledge the support of the Office of Naval Research (Global Award No. N62909-15-1-2003) and KAUST competitive research funding for the work at the King Abdullah University of Science and Technology. We thank Dr. Sean M. Ryno for many useful discussions.
${ }^{1}$ N. Sato, H. Inokuchi, and E. A. Silinsh, Chem. Phys. 115, 269-277 (1987).

${ }^{2}$ S. M. Ryno, M. K. Ravva, X. Chen, H. Li, and J.-L. Brédas, Adv. Energy Mater. 7, 1601370 (2017).

${ }^{3}$ J. Li, G. D'Avino, I. Duchemin, D. Beljonne, and X. Blase, J. Phys. Chem. Lett. 7, 2814 (2016).

${ }^{4}$ S. Verlaak and P. Heremans, Phys. Rev. B 75, 115127 (2007).

${ }^{5}$ G. D' Avino, L. Muccioli, C. Zannoni, D. Beljonne, and Z. G. Soos, J. Chem. Theory Comput. 10, 4959 (2014).

${ }^{6}$ J. W. Ponder, C. Wu, P. Ren, V. S. Pande, J. D. Chodera, M. J. Schnieders, I. Haque, D. L. Mobley, D. S. Lambrecht, R. A. DiStasio, M. Head-Gordon, G. N. I. Clark, M. E. Johnson, and T. Head-Gordon, J. Phys. Chem. B 114, 2549 (2010).

${ }^{7}$ S. M. Ryno, S. R. Lee, J. S. Sears, C. Risko, and J. L. Bredas, J. Phys. Chem. C 117, 13853 (2013).

${ }^{8}$ J. E. Norton and J. L. Bredas, J. Am. Chem. Soc. 130, 12377 (2008).

${ }^{9}$ P. Friederich, V. Meded, F. Symalla, M. Elstner, and W. Wenzel, J. Chem. Theory Comput. 11, 560 (2015).

${ }^{10}$ F. Castet, P. Aurel, A. Fritsch, L. Ducasse, D. Liotard, M. Linares, J. Cornil, and D. Beljonne, Phys. Rev. B 77, 115210 (2008).

${ }^{11}$ S. M. Ryno, Y.-T. Fu, C. Risko, and J.-L. Brédas, ACS Appl. Mater. Interfaces 8, 15524 (2016).

${ }^{12}$ X. Y. Zhu, J. Phys. Chem. Lett. 5, 2283 (2014).

${ }^{13}$ D. Cahen and A. Kahn, Adv. Mater. 15, 271 (2003).

${ }^{14}$ X.-Y. Zhu and A. Kahn, MRS Bull. 35, 443-448 (2010).

${ }^{15}$ N. Ando, M. Mitsui, and A. Nakajima, J. Chem. Phys. 127, 234305 (2007).

${ }^{16}$ J. K. Song, N. K. Lee, J. H. Kim, S. Y. Han, and S. K. Kim, J. Chem. Phys. 119, 3071 (2003).

${ }^{17}$ J. K. Song, N. K. Lee, and S. K. Kim, Angew. Chem., Int. Ed. 42, 213 (2003).

${ }^{18}$ N. Ando, M. Mitsui, and A. Nakajima, J. Chem. Phys. 128, 154318 (2008).

${ }^{19}$ H. Yoshida, K. Yamada, J. Y. Tsutsumi, and N. Sato, Phys. Rev. B 92, 075145 (2015).

${ }^{20}$ M.-C. Kim, E. Sim, and K. Burke, J. Chem. Phys. 134, 171103 (2011).

${ }^{21}$ N. Sai, P. F. Barbara, and K. Leung, Phys. Rev. Lett. 106, 226403 (2011).

${ }^{22}$ A. V. Marenich, S. V. Jerome, C. J. Cramer, and D. G. Truhlar, J. Chem. Theory Comput. 8, 527 (2012).

${ }^{23}$ A. Ruzsinszky, J. P. Perdew, G. I. Csonka, O. A. Vydrov, and G. E. Scuseria, J. Chem. Phys. 125, 194112 (2006).

${ }^{24}$ P. Mori-Sánchez, A. J. Cohen, and W. Yang, J. Chem. Phys. 125, 201102 (2006).

${ }^{25}$ A. J. Cohen, P. Mori-Sánchez, and W. Yang, Chem. Rev. 112, 289 (2011).

${ }^{26}$ T. Körzdörfer, J. S. Sears, C. Sutton, and J. L. Bredas, J. Chem. Phys. 135, 204107 (2011).

${ }^{27}$ C. Sutton, T. Körzdörfer, V. Coropceanu, and J.-L. Brédas, J. Phys. Chem. C 118, 3925 (2014).

${ }^{28}$ T. Körzdörfer, R. M. Parrish, J. S. Sears, C. D. Sherrill, and J. L. Bredas, J. Chem. Phys. 137, 124305 (2012).

${ }^{29}$ J.-D. Chai and M. Head-Gordon, Phys. Chem. Chem. Phys. 10, 6615 (2008).

${ }^{30}$ R. Baer, E. Livshits, and U. Salzner, Annu. Rev. Phys. Chem. 61, 85 (2010).

${ }^{31}$ T. Yanai, D. P. Tew, and N. C. Handy, Chem. Phys. Lett. 393, 51 (2004).

${ }^{32}$ R. Baer and D. Neuhauser, Phys. Rev. Lett. 94, 043002 (2005).

${ }^{33}$ O. A. Vydrov, J. Heyd, A. V. Krukau, and G. E. Scuseria, J. Chem. Phys. 125, 074106 (2006).

${ }^{34}$ K. D. Jordan and P. D. Burrow, Chem. Rev. 87, 557 (1987).

${ }^{35}$ R. B. Campbell, J. M. Robertson, and J. Trotter, Acta Crystallogr. 14, 705 (1961).

36 J. M. Robertson, Rev. Mod. Phys. 30, 155 (1958).

${ }^{37}$ H. C. Alt and J. Kalus, Acta Crystallogr., Sect. B: Struct. Crystallogr. Cryst. Chem. 38, 2595 (1982).

${ }^{38}$ W. L. Jorgensen, D. S. Maxwell, and J. Tirado-Rives, J. Am. Chem. Soc. 118, 11225 (1996).

${ }^{39}$ B. Hess, C. Kutzner, D. van der Spoel, and E. Lindahl, J. Chem. Theory Comput. 4, 435 (2008).

${ }^{40}$ U. Essmann, L. Perera, M. L. Berkowitz, T. Darden, H. Lee, and L. G. Pedersen, J. Chem. Phys. 103, 8577 (1995).

${ }^{41}$ B. Wang, S. L. Li, and D. G. Truhlar, J. Chem. Theory Comput. 10, 5640 (2014).

${ }^{42}$ B. Wang and D. G. Truhlar, J. Chem. Theory Comput. 9, 1036 (2013).

${ }^{43}$ W. J. Hehre, R. Ditchfield, and J. A. Pople, J. Chem. Phys. 56, 2257 (1972).

${ }^{44}$ M. J. Frisch, G. W. Trucks, H. B. Schlegel, G. E. Scuseria, M. A. Robb, J. R. Cheeseman, G. Scalmani, V. Barone, G. A. Petersson, H. Nakatsuji, X. Li, M. Caricato, A. Marenich, J. Bloino, B. G. Janesko, R. Gomperts, B. Mennucci, H. P. Hratchian, J. V. Ortiz, A. F. Izmaylov, J. L. Sonnenberg, D. Williams-Young, F. Ding, F. Lipparini, F. Egidi, J. Goings, B. Peng, 
A. Petrone, T. Henderson, D. Ranasinghe, V. G. Zakrzewski, J. Gao, N. Rega, G. Zheng, W. Liang, M. Hada, M. Ehara, K. Toyota, R. Fukuda, J. Hasegawa, M. Ishida, T. Nakajima, Y. Honda, O. Kitao, H. Nakai, T. Vreven, K. Throssell, J. A. Montgomery, Jr., J. E. Peralta, F. Ogliaro, M. Bearpark, J. J. Heyd, E. Brothers, K. N. Kudin, V. N. Staroverov, T. Keith, R. Kobayashi, J. Normand, K. Raghavachari, A. Rendell, J. C. Burant, S. S. Iyengar, J. Tomasi, M. Cossi, J. M. Millam, M. Klene, C. Adamo, R. Cammi, J. W. Ochterski, R. L. Martin, K. Morokuma, O. Farkas, J. B. Foresman, and D. J. Fox, Gaussian 09, Revision D.01, Gaussian, Inc., Wallingford, CT, 2013.

${ }^{45}$ N. G. Martinelli, J. Ide, R. S. Sanchez-Carrera, V. Coropceanu, J. L. Bredas, L. Ducasse, F. Castet, J. Cornil, and D. Beljonne, J. Phys. Chem. C 114, 20678 (2010).

${ }^{46}$ N. R. Tummala, Z. Zheng, S. G. Aziz, V. Coropceanu, and J.-L. Brédas, J. Phys. Chem. Lett. 6, 3657 (2015).

${ }^{47}$ J. Schiedt and R. Weinkauf, Chem. Phys. Lett. 266, 201 (1997).
${ }^{48}$ L. Crocker, T. Wang, and P. Kebarle, J. Am. Chem. Soc. 115, 7818 (1993).

${ }^{49}$ R. Dennington, T. Keith, and J. Millam, GaussView, Version 5, Semichem Inc., Shawnee Mission, KS, 2009.

${ }^{50}$ M. Mitsui, S. Kokubo, N. Ando, Y. Matsumoto, A. Nakajima, and K. Kaya, J. Chem. Phys. 121, 7553 (2004).

${ }^{51}$ S. M. Ryno, C. Risko, and J.-L. Brédas, J. Am. Chem. Soc. 136, 6421 (2014).

${ }^{52}$ N. Karl, Synth. Met. 133-134, 649 (2003).

${ }^{53}$ V. Podzorov, E. Menard, A. Borissov, V. Kiryukhin, J. A. Rogers, and M. E. Gershenson, Phys. Rev. Lett. 93, 086602 (2004).

${ }^{54}$ S.-i. Machida, Y. Nakayama, S. Duhm, Q. Xin, A. Funakoshi, N. Ogawa, S. Kera, N. Ueno, and H. Ishii, Phys. Rev. Lett. 104, 156401 (2010).

${ }^{55}$ N. Koch, A. Vollmer, I. Salzmann, B. Nickel, H. Weiss, and J. P. Rabe, Phys. Rev. Lett. 96, 156803 (2006).

${ }^{56}$ R. C. Hatch, D. L. Huber, and H. Höchst, Phys. Rev. Lett. 104, 047601 (2010). 